\title{
Lipid-Coated Hybrid Nanoparticles for Enhanced Bacterial Biofilm Penetration and Antibiofilm Efficacy
}

Hiang Wee Lee

Nanyang Technological University

Sharad Kharel

Nanyang Technological University

Say Chye Joachim Loo ( $\sim$ joachimloo@ntu.edu.sg )

Nanyang Technological University

\section{Research Article}

Keywords: Lipid-coated hybrid nanoparticles, biofilm infection, vancomycin, drug delivery,

Staphylococcus aureus, antibiotic resistance

Posted Date: February 22nd, 2022

DOI: https://doi.org/10.21203/rs.3.rs-1301745/v2

License: (a) (1) This work is licensed under a Creative Commons Attribution 4.0 International License.

Read Full License 


\section{Abstract}

Background: The biofilm way of life is a common strategy for bacteria to adapt and gain tolerance towards antibiotic treatments. Up to $80 \%$ of all infections are biofilm-mediated and they are often challenging to treat as the underlying bacterial cells can become $100-1000$-fold more tolerant towards antibiotics. Antibiotic-loaded nanoparticles have gained traction as a potential drug delivery system to treat biofilm infections. Specifically, lipid-coated hybrid nanoparticles (LCHNPs) were investigated on their capability to deliver antibiotics into biofilms. In this study, LCHNPs composed of a poly(lactic-co-glycolic acid) (PLGA) core and dioleoyl-3-trimethylammonium propane (DOTAP) lipid shell were developed and loaded with vancomycin (Van). In vitro antibacterial and antibiofilm tests were performed to evaluate the antimicrobial efficacy of the LCHNPs.

Results: LCHNPs were successfully fabricated with high vancomycin encapsulation efficiency of $48.66 \%$ and loading efficiency of $72.99 \mu \mathrm{g} / \mathrm{mg}$. DOTAP was determined to be successfully coated on the PLGA core by measuring the $\zeta$-potential of the nanoparticles. LCHNPs had a positive $\zeta$-potential of $+36.13 \mathrm{mV}$ which contrasted significantly from the $\zeta$-potential of $-36.83 \mathrm{mV}$ of bare PLGA nanoparticles (PLGANPs). LCHNPs exhibited enhanced antibacterial effects against planktonic Staphylococcus aureus USA300 cells, with at least 6-fold reduction in minimum inhibitory concentration when compared against Free-Van and Van-PLGANPs. When used to treat USA300 biofilms, Van-LCHNPs eradicated up to $99.99 \%$ of the underlying biofilm cells, an effect which was not observed for Free-Van and Van-PLGANPs. Finally, we showed that by possessing a robust DOTAP shell, LCHNPs were able to penetrate deeply into the biofilms.

Conclusion: LCHNPs were shown to display remarkable antimicrobial efficacy towards both planktonic and biofilm cells as the presence of the lipid shell enhanced the interactions with bacterial cells and penetration into biofilms. More work could be done to understand nanoparticle-biofilm interactions; this would help to optimize the LCHNPs further to treat biofilm infections successfully.

\section{Background}

Antibiotic resistant infections are emerging as one of the most critical public health challenges today. In a report published by the Centre for Disease Control and Prevention (CDC) in 2019, it was estimated that 2.8 million antibiotic-resistant infections and more than 35,000 deaths were occurring each year in the United States. The number of infections is expected to increase to 10 million annually by 2050 . It should be realized that infections that are impossible to treat are no longer a distant threat - it has already become a reality [1]. While most antibiotic-resistant infections are still treatable today, they often necessitate the usage of second- or third-line antibiotic treatments which often induce side effects that may prolong care and recovery for patients [2].

The biofilm way of life has been identified as a key strategy employed by bacteria to become more tolerant towards antibiotics; in fact, it was estimated that $65-80 \%$ of all infections were biofilmmediated, highlighting the enormous clinical impact of biofilms on society [3]. Biofilms are generally 
represented as an immobile population of bacteria cells embedded within a matrix that is attached to a surface. The matrix is formed by extracellular polymeric substances (EPS) that are secreted by the bacteria cells. When bacteria, such as Staphylococcus aureus (S. aureus), enter the biofilm state, they become capable of withstanding hostile environments, such as starvation and antibiotic treatments, and cause chronic diseases [4]. Upon entering the biofilm state, the underlying bacteria cells could tolerate antibiotic concentrations up to $100-1000$ times the concentrations typically used to eradicate planktonic bacteria $[5,6]$. Apart from acting as a diffusion barrier to antibiotics, certain components in the EPS could deactivate antibiotics, rendering them ineffective [7]. As a consequence of the inability of existing antibiotics to properly eradicate biofilms, biofilm-associated infections tend to be recurring and life-threatening.

Methicillin-resistant $S$. aureus (MRSA) remains as a leading cause of death and morbidity due to infection, despite many years of infection control efforts [8]. In fact, MRSA strains have been identified as an urgent threat to public health by the US Centers for Disease Control and Prevention (CDC) [9]. Listed as one of the tough-to-treat ESKAPE pathogens, MRSA is ill-famed for causing recurring, long-term skin wound and soft tissue infections in the clinical setting $[10,11]$. To complicate treatments further, MRSA can form sturdy biofilms at infection sites, rendering several antibiotic treatments ineffective. Thus, there is a serious and urgent need to develop alternative treatments to overcome the various mechanisms employed by bacteria biofilms to render current antibiotics ineffective.

The use of nanoparticulate antibiotic delivery systems has been widely explored and showed promising therapeutic effects against bacterial cells in various modes of living. Specifically, lipid-coated hybrid nanoparticles (LCHNPs) have garnered interest as a promising antibiotic delivery system. These particles adopt a core-shell structure, with the core often being an organic polymer surrounded by a lipid shell. By merits of the lipid shell, LCHNPs are postulated to have enhanced affinity towards bacterial cells, resulting in enhanced antibacterial effects [12]. Our prior study had shown that when antibiotics were encapsulated in LCHNPs, there was a reduction in minimum inhibitory concentration (MIC) of certain antibiotics by as much as 32 -fold when the LCHNPs were used to treat planktonic cells [7]. In a more recent study, LCHNPs loaded with ampicillin were used to treat protozoa infected with Enterococcus faecalis in an intracellular infection model. When compared to unencapsulated ampicillin, the ampicillinloaded LCHNPs were able to boost the survivability of protozoa cells by up to $400 \%$ while also significantly reducing the quantity of intracellular bacterial cells [13]. These studies explored the potential of LCHNPs in treating complex bacterial infections, while also highlighting the unexplored potential of LCHNPs in treating biofilms.

In this study, we investigated the potential of LCHNPs in treating MRSA-USA300 biofilms. It was hypothesized that the presence of a cationic lipid coating in LCHNPs helps to enhance the penetration of these nanoparticles into biofilms, resulting in enhanced antibiofilm effects. Specifically, we optimized the fabrication of vancomycin-loaded LCHNPs with PLGA core and DOTAP shell through a simple, double emulsion solvent evaporation approach. The resulting nanoparticles were determined to have high encapsulation and loading efficiencies for vancomycin, despite it being a hydrophilic antibiotic. 
Furthermore, antibacterial tests showed that the vancomycin-loaded LCHNPs exhibited enhanced antibacterial and antibiofilm effects when compared to free vancomycin and non-lipid-coated, vancomycin-loaded PLGA nanoparticles. Finally, we verified our hypothesis by performing a biofilm penetration study with LCHNPs of various DOTAP:PLGA ratios. Through this, we concluded that the presence of a robust lipid layer exhibiting a strong positive charge is required to achieve enhanced penetration into USA300 biofilms. In summary, this study highlights the ability of LCHNPs to eradicate bacterial biofilms, and the potential to be extended to other biofilm models.

\section{Results And Discussion}

Fabrication and optimization of LCHNPs. In this study, LCHNPs were prepared using a simple emulsification technique. LCHNPs could be thought to consist of a polymeric PLGA core surrounded by a layer of lipid, DOTAP. By varying the DOTAP-to-PLGA ratio, LCHNPs with various EE, LE and ל-potentials could be achieved (Table 1).

From Table 1, several observations could be drawn on the effects of the masses of PLGA and DOTAP used on the characteristics of the resulting LCHNPs. A comparison between LCHNP60:10 and LCHNP120:10 highlighted that a higher quantity of PLGA used during fabrication was associated with an increase in vancomycin EE, and a decrease in LE and $\zeta$-potential. The increase in EE from 26.12-44.19\% with higher PLGA quantity was consistent with several published studies. These studies stated that an increase in PLGA quantity used during fabrication led to an increase in the core size of the nanoparticles, allowing for more vancomycin molecules to be encapsulated $[14,15]$. A decrease in LE from $49.34 \mu \mathrm{g} / \mathrm{mg}$ to $40.79 \mu \mathrm{g} / \mathrm{mg}$ was observed when a higher quantity of PLGA was used. With an increase in the nanoparticle core size, there is also an increase in the mass of each NP, which likely explains the reduction of LE since LE is inversely proportional to the mass of nanoparticles. Also, the stronger negative

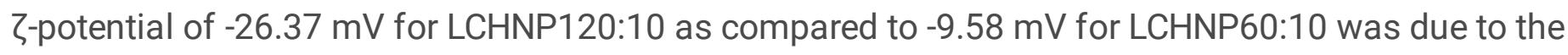
higher quantity of PLGA used in LCHNP120:10. This suggested that there was insufficient DOTAP to coat the PLGA core adequately. The EE and LE of LCHNP120:20 were reduced when compared to LCHNP60:20; this appeared to be an anomaly as the EE was expected to increase when more PLGA was used. LCHNP120:20 was visually observed to be a sticky paste instead of a white powder like the other samples; it was postulated that the high quantities of PLGA and DOTAP used during the fabrication of LCHNP120:20 led to an increased viscosity of the overall emulsion, and thus resulted in poor fabrication of the nanoparticles.

A comparison between LCHNP60:10 and LCHNP60:20 revealed that the higher mass of DOTAP used in LCHNP60:20 was associated with an increase in EE, LE and Z-potential. Baek et al. suggested that hydrophilic drugs, such as vancomycin, tend to localize on the surface of hydrophobic PLGA nanoparticles. By incorporating a layer of lipid onto PLGA nanoparticles, hydrophilic drugs could be trapped between the PLGA core and lipid layer, resulting in increased EE and LE [7]. Thus, in the case of LCHNP60:20, a higher quantity of DOTAP used could lead to a more robust lipid layer, as verified by the 
strong, positive $\zeta$-potential. Consequently, the robust layer of DOTAP contributed to the trapping of hydrophilic vancomycin molecules within the LCHNP, resulting in higher EE and LE.

Amongst LCHNP60:10, LCHNP 60:20, LCHNP120:10, and LCHNP120:20, it was observed that LCHNP60:20 displayed desirable properties required for the delivery of vancomycin into biofilms, whereby: 1) a high value of LE signifying that more antibiotics could be delivered with smaller quantity of LCHNPs; 2 ) a high value of EE indicating that a higher proportion of antibiotic used during fabrication was encapsulated, resulting in lesser wastage; and 3) a positive surface charge which would be beneficial for penetrating into biofilms. Thus, further optimization was carried out based on the formulation for LCHNP60:20, which led to LCHNP45:20 and LCHNP30:20.

It was noted that LCHNP45:20 had the highest EE, LE and Z-potential of $48.66 \%, 72.99 \mu \mathrm{g} / \mathrm{mg}$ and +36.13 $\mathrm{mV}$ respectively (Figure 1a-c). These measurements were significantly higher than those of LCHNP60:20 and LCHNP30:20. In fact, the LE of the optimized LCHNP45:20 were significantly higher than the widely reported values of $2.6-15.8 \mu \mathrm{g} / \mathrm{mg}$ for reported nanoparticulated systems used to encapsulate hydrophilic molecules [16-20]. Thus, the formulation for LCHNP45:20 was chosen for subsequent experiments.

Physical characterization of optimized LCHNPs and comparison with PLGANPs. To determine the role of DOTAP in the nanoparticulate systems, bare and vancomycin-loaded PLGANPs were fabricated using the exact same parameters as those used for the fabrication of the optimized LCHNPs, less DOTAP. Through dynamic light scattering, the hydrodynamic diameters of both PLGANPs and LCHNPs were determined to be $221.03 \pm 6.50 \mathrm{~nm}$ and $207.83 \pm 2.06 \mathrm{~nm}$ respectively (Figure $2 \mathrm{a}$ ). The sizes of these nanoparticles fall within the desirable range of $100-500 \mathrm{~nm}$ for penetration into biofilms through water channels and EPS without eliciting rapid clearance by the host [21]. LCHNPs were observed to be significantly smaller (**) than the bare PLGANPs. This was likely due to the presence of the cationic DOTAP, which played a crucial role in regulating the size of LCHNPs, preventing the coalescence of these nanoparticles. The drastic difference in $\zeta$-potentials of PLGANPs and LCHNPs $(-36.83 \pm 1.24 \mathrm{mV}$ and $36.13 \pm 0.31 \mathrm{mv}$ respectively) suggested that DOTAP was successfully coated onto the PLGA core of LCHNPs. The FESEM images (Figures $2 \mathrm{~b} \& 2 \mathrm{c}$ ) showed the spherical morphologies of both PLGANPs and LCHNPs. The coreshell structure of LCHNPs was further verified using TEM (Figure 2d).

The EE and LE values of LCHNPs were significantly improved over those of PLGANPs. The addition of the DOTAP layer on the PLGA surface possibly prevented vancomycin molecules from escaping out of the nanoparticles during fabrication, leading to higher EE and LE. Given that vancomycin is highly hydrophilic, there is a high tendency of it escaping from the oil phase of the emulsion into the bulk aqueous phase during the fabrication process. This could be observed from the lower EE and LE values of PLGANPs of $24.11 \pm 0.64 \%$ and $52.25 \pm 1.38 \mu \mathrm{g} / \mathrm{mg}$ respectively. On the other hand, LCHNPs had an EE of $48.66 \pm 0.42 \%$ and LE of $72.99 \pm .63 \mu \mathrm{g} / \mathrm{mg}$, highlighting the essential role of the lipid layer in achieving high EE and LE of hydrophilic molecules in polymeric nanoparticles [22, 23]. Furthermore, the lipid layer was observed to retard the release of vancomycin from the nanoparticles. From the release 
profiles (Figure 2e), 43.93\% of the encapsulated vancomycin within Van-LCHNPs was released, while $92.26 \%$ of the drug was released from Van-PLGANPs. The burst release of vancomycin from both types of nanoparticles within the first 24 hours was not unexpected, given that the hydrophilic vancomycin would tend to localize at the surface of the hydrophobic nanoparticles. Nonetheless, vancomycin release data within the first 24 hours suggested that the presence of the lipid layer mitigated the burst release of vancomycin. This was in good agreement with Sivadasan et al. who suggested that the presence of a lipid shell on nanoparticulate carriers reduced the rate of water penetration, resulting in reduced rate of hydrolysis of the polymeric core and thus, a slower rate of release [23].

Presence of DOTAP layer enhanced antibacterial efficacy against planktonic USA300. Prior studies had demonstrated the effectiveness of positively-charged drug delivery systems, such as cationic liposomes and certain antimicrobial peptides, on making first contact and continuous binding with bacteria [24, 25]. Therefore, it was hypothesized that Van-LCHNPs, being positively charged, would have an enhanced therapeutic effect against planktonic USA300 compared to bare Van-PLGANPs. Comparing against FreeVan, it was observed that Van-LCHNPs exhibited enhanced antibacterial effects against planktonic USA300 (Table 2). By loading vancomycin into LCHNPs, there was a reduction in MIC and MBC by at least six-fold as compared to the MIC and MBC values for Free-Van. This contrasted with the case for Van-PLGANPs whereby MIC and MBC values increased. PLGANPs have poor interactions with the USA300 cells due to the negative charge on both. Arakha et al. reported that negatively-charged nanoparticles do not adhere to bacteria owing to poor electrostatic attraction [26]. Furthermore, the controlled release of vancomycin from Van-PLGANPs implied that inhibitory or biocidal concentrations of vancomycin could not be reached quickly, ultimately resulting in higher MIC and MBC values observed as compared to Free-Van. To eliminate the possibility that the enhanced bacterial effects of LCHNPs were caused directly by the intrinsic antibacterial property of the nanoparticles, blank LCHNPs and PLGANPs were utilized as controls; data from these blank runs showed that no antibacterial effects were observed from extremely high nanoparticle concentrations up to $10,000 \mu \mathrm{g} / \mathrm{mL}$. The stronger antibacterial effect of Van-LCHNPs in comparison to Van-PLGANPs could be due to the presence of the cationic DOTAP layer playing a significant role in allowing the LCHNPs to make first contact and continuous binding with the USA300 cells. This is in good agreement with Costa et al. who correlated the positive effect of enhancing interactions between nanoparticles and bacterial cells on antibiotic delivery [27].

\section{Enhanced antibiofilm effect against USA300 biofilms using LCHNPs. To evaluate the capability of}

LCHNPs to eradicate USA300 biofilms, static biofilms were cultivated in 8-wells chambered coverglasses. The cultivated biofilms were then treated overnight with Free-Van, Van-PLGANPs and Van-LCHNPs with vancomycin concentrations ranging from $256 \mu \mathrm{g} / \mathrm{mL}$ to $0.25 \mu \mathrm{g} / \mathrm{mL}$ in 4-fold dilutions. Through crystal violet staining, remnant biofilms following treatment could be visually observed (Figure 3a-c). It was observed that Free-Van and Van-PLGANPs did not cause any significant reduction in biomass across all tested concentrations as compared to the untreated controls. This suggested that both groups were not able to sufficiently eradicate the underlying bacterial cells to cause any biofilm disruption. On the other hand, significant reductions in biomass were observed for Van-LCHNPs, specifically for vancomycin concentrations of $256-4 \mu \mathrm{g} / \mathrm{mL}$. It should be noted that vancomycin concentrations of $256 \mu \mathrm{g} / \mathrm{mL}$ and 
$64 \mu \mathrm{g} / \mathrm{mL}$ for Van-LCHNPs corresponded to higher concentrations of nanoparticles that were used for treatment. The high concentrations led to residual nanoparticles that were not completely removed during the washing step in the protocol which subsequently led to the observed signals following crystal violet staining which should not be incorrectly interpreted as biomass. The observations from crystal violet staining were supported with CFU data that were derived from remnant biofilms. As observed from Figure 3d, Van-LCHNPs exhibited an exceptional capability to eradicate USA300 biofilms across all tested concentrations of vancomycin. At $256 \mu \mathrm{g} / \mathrm{mL}$, Van-LCHNPs showed the highest degree of biofilm cells eradication with 4 logs reduction (or 99.99\%) in CFU/mL as compared to the untreated control $\left(\sim 2 \times 10^{10}\right.$ $\mathrm{CFU} / \mathrm{mL}$ ). Even at $0.25 \mu \mathrm{g} / \mathrm{mL}$ of vancomycin, Van-LCHNPs could reduce the CFU count by 2 logs ( $99 \%$ ). On the other hand, Free-Van and Van-PLGANPs were largely incapable of meaningfully reducing the CFUs counts in USA300 biofilms. This suggested that Free-Van and Van-PLGANPs could not adequately penetrate through the biofilms for vancomycin to be uptake by the underlying bacterial cells. We postulated that the strong antibiofilm capability of Van-LCHNPs is due to the presence of the DOTAP coating which enhanced interactions between the nanoparticles and the biofilm EPS, leading to enhanced penetration. Wang et al. suggested that the utilization of lipids which are chemically similar to bacterial membranes could improve the fusion of nanoparticles with bacterial cells [28]. Furthermore, Sugano et al. reported that cationic liposomes do not only interact well with planktonic bacterial cells, but also biofilms [25]. This was due to electrostatic interactions between the cationic liposomes and biofilm matrix, which is often negatively charged. Consequently, Van-LCHNPs, coated with cationic DOTAP, were able to penetrate deeper into the biofilms to deliver the antibiotics directly to the USA300 cells.

\section{Enhanced antibiofilm capability of LCHNPs could prevent recalcitrant infections. A key challenge in} treating biofilm infections arises from the fact that it is hard for antibiotics or antibiotic delivery system to penetrate deeply into biofilms to eradicate the underlying cells uniformly throughout the entire thickness of the biofilm [28]. Even if killing occurs, biofilm cells may not be sufficiently eradicated, and the biofilm matrix remains intact. This often gives rise to recalcitrant infections as biofilms which are not completely removed often become a starting point for biofilm regrowth [29]. From Figure 3c, it was observed that there were little biomass remaining following treatment with Van-LCHNPs corresponding to $4-256$ $\mu \mathrm{g} / \mathrm{mL}$, suggesting that biofilms were removed. To verify this, confocal microscopy was performed following treatment to image the remaining biofilms. The biofilms were stained with Syto 9 and PI prior to imaging.

Figure 4a shows the confocal microscopy images for each sample group. When biofilms were treated with Free-Van $(0.25-16 \mu \mathrm{g} / \mathrm{mL})$, the confocal images showed that the bulk of the biofilm cells were alive (green signals) even though the tested concentrations exceeded the $\mathrm{MBC}$ of vancomycin required to completely eradicate planktonic USA300 cells. Also, digital measurement of the biofilm thicknesses (Figure $4 \mathrm{~b}$ ) showed that biofilms treated with Free-Van had increased in thickness as compared to the untreated control. The increase in biofilm thickness could be due to the mechanism of action for vancomycin, by interrupting cell wall synthesis resulting in bacterial lysis. As a result, biomolecules leaking from the lysed cells may be retained in the biofilm, leading to an increase in biofilm thickness. For 
Van-PLGANPs, only at concentrations corresponding to $4 \mu \mathrm{g} / \mathrm{mL}$ and $16 \mu \mathrm{g} / \mathrm{mL}$ of vancomycin exhibited any antibacterial effect towards the biofilm cells. The killing of biofilm cells at $4 \mu \mathrm{g} / \mathrm{mL}$ appeared to be closer to the top surface of the biofilm, while biofilm cells residing deeper remained alive. At $16 \mu \mathrm{g} / \mathrm{mL}$, the biofilm cells appeared to be eradicated throughout the thickness of the biofilm. However, the biofilm remained intact and could lead to regrowth of the biofilm. For Van-LCHNPs, the thickness of the biofilms appeared to be significantly reduced across all 4 concentrations that were imaged. The degree of biofilm removal was most pronounced at concentrations of $1 \mu \mathrm{g} / \mathrm{mL}, 4 \mu \mathrm{g} / \mathrm{mL}$ and $16 \mu \mathrm{g} / \mathrm{mL}$. Antibiofilm effects appeared to be dose-dependent, whereby the dead cells appeared to be distributed throughout the remaining thickness of the biofilms for $4 \mu \mathrm{g} / \mathrm{mL}$ and $16 \mu \mathrm{g} / \mathrm{mL}$. Overall, the data suggested that VanLCHNPs possessed excellent antibiofilm ability as it could eradicate the underlying cells and cause biofilm removal.

\section{Presence of a robust coating of DOTAP led to enhanced penetration ability of LCHNPs into USA300}

biofilms. It was verified through several methods that Van-LCHNPs possessed exceptional antibiofilm ability over Free-Van and Van-PLGANPs (Figures 3 and 4). This study stemmed from the hypothesis that the presence of a cationic lipid coating could enhance the penetration of nanoparticles into biofilms, leading to improved antibiofilm capabilities. To verify role of DOTAP coating in enabling enhanced penetration into biofilms, PI-loaded LCHNPs of various DOTAP:PLGA ratios (Figure 5a) were fabricated. These PI-loaded nanoparticles were then incubated overnight with USA300 biofilms prior to confocal microscopy imaging. By utilizing the Z-stack function of the confocal microscope, it was investigated how deep into the biofilms these nanoparticles were able to penetrate.

Confocal images (Figure 5) showed that nanoparticles with DOTAP:PLGA ratio of 0:45 (equivalent to PLGANPS) coating exhibited poor penetration into USA300 biofilms while penetration into biofilms increased gradually as the DOTAP:PLGA ratio was increased from 0:45 to 20:45. This could be observed from the increase in red signals coming from the PI-loaded nanoparticles as the DOTAP:PLGA ratio was increased. This suggested that DOTAP:PLGA ratio and the degree of penetration into biofilms had a doseresponse relationship, implying that the DOTAP coating played a causal role in allowing the LCHNPs to penetrate deeply into the biofilms. To the best knowledge of the authors at the time of writing, this is the first time that a cationic lipid coating is shown to directly enhance the penetrating capability of nanoparticles, especially for LCHNPs, into biofilms.

The penetration of LCHNPs into biofilms could be described using the nanoparticle-biofilm transport phenomena. Firstly, nanoparticles must be transported to the biofilm-fluid interface; secondly, the nanoparticles must attach to the outer surface of the biofilms; lastly, the nanoparticles would have to migrate into the biofilm. Interactions with the biofilm EPS are often governed by the surface properties of the nanoparticles, such as surface charge and functional groups present on the surface. These interactions are critical for nanoparticles to establish first contact (attachment) required with the biofilm surface before the nanoparticles could migrate within the biofilms [30]. In the case of LCHNPs in this study, a strong positively charged surface was presented to the biofilm EPS, allowing for strong interactions between the negatively charged EPS components and the LCHNPS. This contrasts with 
PLGANPs, which present a negatively charged surface and thus, have poor interactions with the biofilm EPS. This observation is congruent with studies that reported that electrostatic interactions played a critical role in determining the ability of nanoparticles to adhere onto biofilm surfaces [31, 32].

\section{Conclusion}

In this study, the fabrication of LCHNPs was optimized, resulting in nanoparticles which demonstrated high LE and EE of vancomycin. The antibacterial and antibiofilm effects of Van-LCHNPs were studied and compared against Free-Van and Van-PLGANPs. In vitro tests against planktonic and biofilm USA300 showed that LCHNPs demonstrated enhanced antimicrobial effects. Specifically, LCHNPs exhibited exceptional antibiofilm ability by killing off biofilm cells and facilitated the removal of biofilm matrix. The removal of biofilm matrix is crucial for preventing recalcitrant infections. Finally, for the first time, we showed that the presence of a cationic lipid layer on LCHNPs directly enhanced the penetrating capability of the nanoparticles into USA300 biofilms. Given that biofilms are extremely complex and dynamic in nature, the interactions of nanoparticles with biofilms are still not well understood. With further investigations into the interactions between nanoparticles and biofilms, LCHNPs could be optimized to further enhance their capability to eradicate biofilms.

\section{Materials And Methods}

Materials. Resomer® RG $502 \mathrm{H}$ poly(D,L-lactide-co-glycolide) (PLGA, MW = 7 - 17 kDa, acid-terminated), poly(vinyl alcohol) (PVA, MW = $30-80 \mathrm{kDa}, 87 \%$ hydrolyzed), Span ${ }^{\circledR} 80$ and vancomycin hydrochloride were purchased from Sigma-Aldrich. 1,2-dioleoyl-3-trimethylammonium-propane (DOTAP, chloride salt) was purchased from Avanti Lipids. Unless stated otherwise, all solvents used are of analytical grade and purchased from Sigma-Aldrich. HPLC-grade solvents used for HPLC mobile phases were purchased from Tedia.

Fabrication and optimization of LCHNPs. Blank LCHNPs were fabricated using an oil-in-water (O/W) single emulsion solvent evaporation technique. The organic phase was prepared by dissolving $45 \mathrm{mg}$ of PLGA and $20 \mu \mathrm{L}$ of Span $\circledast 80$ in $2 \mathrm{~mL}$ of dichloromethane (DCM). The aqueous phase was prepared by adding $20 \mathrm{mg}$ of DOTAP into $20 \mathrm{~mL}$ of $1 \% \mathrm{w} / \mathrm{v}$ PVA solution (in $0.95 \% \mathrm{MES}$ buffer, $\mathrm{pH}$ 6.9). Under probe sonication on an ice bath at $40 \%$ amplitude, the organic phase was added into the aqueous phase to form an emulsion and allowed to sonicate for 1 minute. After probe sonication, the $\mathrm{O} / \mathrm{W}$ emulsion was left to stir at $1000 \mathrm{rpm}$ for 4 hours to evaporate the DCM. Following evaporation, the resulting solution was washed thrice by centrifugation at $18 \mathrm{k} \mathrm{rpm}$ for 12 minutes. After the final wash, the resulting pellet was frozen and lyophilized to obtain LCHNP powder.

To fabricate vancomycin-loaded LCHNPs (Van-LCHNP), a water-in-oil-in-water (W/O/W) double emulsion solvent evaporation approach was utilized. Vancomycin solution was prepared by dissolving vancomycin hydrochloride powder in 1\% w/v PVA (in 0.95\% MES buffer, $\mathrm{pH} 6.9$ ) at a concentration of $24.375 \mathrm{mg} / \mathrm{mL}$. The primary W/O emulsion was formed by adding $400 \mu \mathrm{L}$ of vancomycin solution to the organic phase 
and probe-sonicated at $40 \%$ amplitude for 12 cycles in pulse mode ( 3 seconds on, 2 seconds off). Subsequently, the secondary W/O/W emulsion was obtained by adding the primary emulsion into the secondary aqueous phase and sonicating at $40 \%$ amplitude for 1 minute. Following sonication, the emulsion was allowed to stir at $1000 \mathrm{rpm}$ for 4 hours to evaporate the DCM, and subsequently washed thrice by centrifugation at $18 \mathrm{k} \mathrm{rpm}$ for 12 minutes. The resulting pellet was frozen and lyophilized to obtain Van-LCHNP powder.

For the fabrication of blank and vancomycin-loaded control nanoparticles, DOTAP was removed from the abovementioned formulation to form blank and Van-PLGANP powder.

Characterization of LCHNPs. The hydrodynamic diameters and zeta potentials ( $\zeta$-potential) of LCHNPs were measured by dynamic light scattering using a Malvern Zeta Sizer ZS. Briefly, lyophilized powder of LCHNPs were diluted in deionized $\mathrm{H}_{2} \mathrm{O}$ to obtain $1 \mathrm{mg} / \mathrm{mL}$ suspensions, which were then added into Malvern disposable folded capillary cell (DTS1070) and inserted into the equipment for measurements to be taken. For field-emission scanning electron microscopy (FESEM), the sample was prepared by applying a thin layer of LCHNP dry powder onto carbon tape. This was followed by sputter-coating the layer with Pt for 15 seconds, after which the sample was then imaged under JEOL FESEM $7600 \mathrm{~F}$ at $2 \mathrm{kV}$ accelerating voltage with a probe current of 8 . For transmission electron microscopy (TEM), the LCHNP suspension was added onto a hydrophilic Formvar-coated copper grid for 3 minutes, followed by uranyl acetate staining. The resulting sample was then imaged using Carl Zeiss Libra 120 Plus.

Vancomycin loading measurement. Ten milligrams of lyophilized Van-PLGANP and Van-LCHNP powder were dissolved in $1 \mathrm{~mL}$ of dimethyl sulfoxide (DMSO) and filtered through a $0.22 \mu \mathrm{m}$ pore size syringe filter. High-performance liquid chromatography (HPLC) with a UV-Vis detector was used to detect and measure the quantity of vancomycin in the solution. The analyte was passed through a C18 column (Zorbax SB, $5.0 \mu \mathrm{m}, 4.6 \times 250 \mathrm{~mm}$ ) and the absorbance at $282 \mathrm{~nm}$ was measured for the detection of vancomycin. In the presence of an absorption peak, the peak area was correlated to the concentration of vancomycin in the analyte using a simple linear regression model with the appropriate standard solutions. To calculate encapsulation efficiency (EE\%) and loading efficiency (LE), the following equations were used for calculation:

$$
\begin{gathered}
\mathrm{EE} \backslash \%=\frac{\text { Mass of antibiotic encapsulated }}{\text { Total mass of antibiotic used in fabrication }} \times 100 \backslash \% \\
L E(\mu \mathrm{g} / \mathrm{mg})=\frac{\text { Massofantibioticencapsulated }}{\text { MassofLCHNPused }}
\end{gathered}
$$

In vitro drug release. In vitro release study was performed using dialysis technique. Briefly, $20 \mathrm{mg}$ of sample powder were suspended in $5 \mathrm{~mL}$ of tryptic soy broth (TSB) and transferred into a dialysis bag (3 $\mathrm{kDa}$ cut-off). The dialysis bag was then immersed in $50 \mathrm{~mL}$ of TSB and allowed to stir at $200 \mathrm{rpm}$. Two millilitres of release medium were collected at pre-determined timepoints and replaced with fresh TSB medium. The amount of antibiotic released was measured using HPLC UV-Vis. 
Bacterial strain and growth conditions. Methicillin-resistant Staphylococcus aureus USA300 strain was used as the model pathogen in this study. A defrost glycerol stock of USA300 was plated on tryptic soy agar (TSA) and incubated overnight at $37^{\circ} \mathrm{C}$ to obtain single colonies. Prior to each experiment, a single colony was inoculated into $10 \mathrm{~mL}$ of fresh TSB and incubated overnight at $37^{\circ} \mathrm{C}$.

Antibiotic susceptibility test on planktonic USA300. The minimum inhibitory concentration (MIC) and minimum biocidal concentration (MBC) were determined using the broth dilution method adapted from Wiegand et al. [33]. Briefly, the overnight culture of USA300 was washed thrice with PBS using centrifugation ( $8,000 \mathrm{rpm}, 5$ minutes), and diluted to an optical density of 0.2 at $600 \mathrm{~nm}$ in TSB. This corresponds to $1 \times 10^{8} \mathrm{CFU} / \mathrm{mL}$ of planktonic USA300 cells. The sample groups tested in this experiment include free vancomycin (Free-Van), Van-PLGANP and Van-LCHNP. In sterile 96-well plates, two-fold serial dilutions were performed for each sample group such that the concentrations of vancomycin, both free and released from nanoparticles, ranged from $0.25-128 \mu \mathrm{g} / \mathrm{mL}$. Finally, $100 \mu \mathrm{L}$ of the diluted bacterial culture were added into each well, resulting in a final bacterial concentration of about $5 \times 10^{7} \mathrm{CFU} / \mathrm{mL}$. Blank PLGANP and LCHNP were also tested in concentrations that were significantly higher than VanPLGANP and Van-LCHNP to determine if these control particles possessed any intrinsic antibacterial properties. Controls containing only bacteria (untreated) or medium without cells (blank) were also tested. The 96-well plates were then incubated overnight in a $37^{\circ} \mathrm{C}, 5 \% \mathrm{CO}_{2}$ incubator. Following incubation, the optical densities at $600 \mathrm{~nm}$ of each well were measured using Tecan Microplate Reader M200.

Subsequently, selected wells were plated onto TSA to determine the MBC. The MIC was defined as the lowest concentration of vancomycin which prohibits planktonic bacterial growth, corresponding to an optical density difference of less than $10 \%$ from the control. The MBC was defined as the lowest concentration of vancomycin required to completely eradicate the USA300 planktonic cells as observed from the TSA plates.

In vitro antibiofilm test. The abilities of Free-Van, Van-PLGANP and Van-LCHNP to eradicate biofilm cells were evaluated in this study. To culture biofilms, an overnight culture of USA300 was washed thrice with PBS (8,000 rpm, 5 minutes) and diluted in TSB supplemented with $1 \% \mathrm{w} / \mathrm{v}$ glucose and $50 \mu \mathrm{g} / \mathrm{mL}$ of methicillin. The addition of $1 \% \mathrm{w} / \mathrm{v}$ glucose and $50 \mu \mathrm{g} / \mathrm{mL}$ methicillin were necessary to form robust biofilms capable of withstanding the multiple washing steps during the experiments. $500 \mu \mathrm{L}$ of the diluted culture $\left(\mathrm{OD}_{600}=0.4\right)$ were added into each well of an 8-well chambered coverglass (Nunc ${ }^{\mathrm{TM}}$ Lab$\mathrm{Tek}^{\mathrm{TM}}$ II) and allowed to incubate overnight in a $37^{\circ} \mathrm{C}, 5 \% \mathrm{CO}_{2}$ incubator. Following incubation, the supernatant in each well was disposed and each well was washed once with PBS to remove any residual planktonic cells. This was followed by adding $500 \mu \mathrm{L}$ of Free-Van or equivalent concentrations of NP suspensions into each well, ranging from $0.25-256 \mu \mathrm{g} / \mathrm{mL}$ of vancomycin in four-fold serial dilutions. Once the necessary treatment and control groups were added, the chambered coverglasses were incubated overnight. Following treatment, the supernatant in each well was disposed and washed once with PBS to remove residual planktonic cells and nanoparticles, following which different characterization methods were employed to observe the antibiofilm efficacies of each treatment group. First, for post-treatment visual observations of the remaining biofilms, if any, $500 \mu \mathrm{L}$ of $0.1 \% \mathrm{v} / \mathrm{v}$ crystal 
violet was added into each well. The wells were allowed to sit for 15 minutes for crystal violet to stain the biofilms, after which the crystal violet supernatant was disposed, and each well was washed twice with PBS. Second, to observe if any killing of biofilm cells took place throughout the biofilms, Syto9 and propidium iodide $(\mathrm{PI})$ from the LIVE/DEAD ${ }^{\mathrm{TM}}$ BacLight $^{\mathrm{TM}}$ bacterial viability kit were used to stain the biofilm cells following treatment. The stained biofilms were then imaged using confocal scanning laser microscopy (CLSM, Zeiss LSM780). Excitation lasers were $442 \mathrm{~nm}$ for Syto9-labelled cells and $561 \mathrm{~nm}$ for PI-labelled cells. Finally, to determine the CFU reduction for each treatment group, any residual biofilms in the wells post-treatment were resuspended in TSB through rigorous pipetting. The suspensions were then plated onto TSA plates and incubated overnight, after which the CFU reduction could be determined.

Determining biofilm-penetrating capabilities of LCHNPs. The ability of LCHNPs to penetrate biofilms were evaluated using CLSM. Instead of antibiotics, each LCHNPs group was loaded with PI. To determine the effect of the lipid layer on biofilm penetration, PI-loaded LCHNPs, with various DOTAP-to-PLGA ratios, were fabricated. To prepare the specimens for CLSM imaging, $2.5 \mathrm{mg} / \mathrm{mL}$ of the PI-loaded NP suspensions were added into biofilm-cultivated wells and allowed to incubate overnight. This was followed by washing off any loose nanoparticles with PBS, and staining the biofilms with Syto9, prior to CLSM imaging.

\section{Declarations}

Acknowledgements: The authors would like to acknowledge the Advanced Biofilm Imaging Facility (ABIF) at SCELSE for providing the Zeiss LSM780 CLSM platform used in this study, and especially Dr Radek Machán and Dr Yong Hwee Foo for rendering the technical assistance required.

Authors' contributions: HWL designed the work, performed experiments, analysed data, and wrote the original draft. SK performed transmission electron microscopy, analysed the associated data, and revised the draft. SCJL supervised the work and revised the draft. All authors read and approved the final manuscript.

Competing interests: The authors declare that they have no competing interests.

Data availability: All data generated or analysed during this study are included in this published article.

Ethics declaration: Not applicable.

Consent for Publication: Not applicable.

Funding: This project was supported by funding from the Singapore Centre of Environmental Life Sciences Engineering (SCELSE) (MOE/RCE: M4330019.C70), Ministry of Education AcRF-Tier 1 grant (RG19/18 and RT08/19), the Singapore National Biofilm Consortium (SNBC/2021/SF2/P04) and the Singapore Food Agency (SFS_RND_SUFP_001_06). 


\section{References}

1. Antibiotic resistance threats in the United States, 2019. 2019.

2. O'Neill J: Tackling drug-resistant infections globally: final report and recommendations. 2016.

3. Joo HS, Otto M: Molecular basis of in vivo biofilm formation by bacterial pathogens.Chem Biol 2012, 19:1503-1513.

4. Roy R, Tiwari M, Donelli G, Tiwari V: Strategies for combating bacterial biofilms: A focus on antibiofilm agents and their mechanisms of action.Virulence 2018, 9:522-554.

5. Olivares E, Badel-Berchoux S, Provot C, Prévost G, Bernardi T, Jehl F: Clinical Impact of Antibiotics for the Treatment of Pseudomonas aeruginosa Biofilm Infections.Frontiers in Microbiology 2020, 10.

6. Wille J, Coenye T: Biofilm dispersion: The key to biofilm eradication or opening Pandora's box? Biofilm 2020, 2:100027.

7. Baek J-S, Tan CH, Ng NKJ, Yeo YP, Rice SA, Loo SCJ: A programmable lipid-polymer hybrid nanoparticle system for localized, sustained antibiotic delivery to Gram-positive and Gram-negative bacterial biofilms.Nanoscale Horizons 2018, 3:305-311.

8. Li J, Zhong W, Zhang K, Wang D, Hu J, Chan-Park MB: Biguanide-Derived Polymeric Nanoparticles Kill MRSA Biofilm and Suppress Infection In Vivo.ACS Applied Materials \& Interfaces 2020, 12:2123121241.

9. Gajdács M: The Continuing Threat of Methicillin-Resistant Staphylococcus aureus.Antibiotics (Basel) 2019, 8.

10. Ma Y-X, Wang C-Y, Li Y-Y, Li J, Wan Q-Q, Chen J-H, Tay FR, Niu L-N: Considerations and Caveats in Combating ESKAPE Pathogens against Nosocomial Infections.Advanced science (Weinheim, BadenWurttemberg, Germany) 2019, 7:1901872-1901872.

11. De Oliveira DMP, Forde BM, Kidd TJ, Harris PNA, Schembri MA, Beatson SA, Paterson DL, Walker MJ: Antimicrobial Resistance in ESKAPE Pathogens.Clinical microbiology reviews 2020, 33:e0018100119.

12. Jiang L, Lee HW, Loo SCJ: Therapeutic lipid-coated hybrid nanoparticles against bacterial infections.RSC Advances 2020, 10:8497-8517.

13. Tan CH, Jiang L, Li W, Chan SH, Baek J-S, Ng NKJ, Sailov T, Kharel S, Chong KKL, Loo SCJ: LipidPolymer Hybrid Nanoparticles Enhance the Potency of Ampicillin against Enterococcus faecalis in a Protozoa Infection Model.ACS Infectious Diseases 2021, 7:1607-1618.

14. Ismail R, Sovány T, Gácsi A, Ambrus R, Katona G, Imre N, Csóka I: Synthesis and Statistical Optimization of Poly (Lactic-Co-Glycolic Acid) Nanoparticles Encapsulating GLP1 Analog Designed for Oral Delivery.Pharmaceutical Research 2019, 36:99.

15. Ramalho MJ, Loureiro JA, Coelho MAN, Pereira MC: Factorial Design as a Tool for the Optimization of PLGA Nanoparticles for the Co-Delivery of Temozolomide and 06-Benzylguanine.Pharmaceutics 2019, 11:401. 
16. Seedat N, Kalhapure RS, Mocktar C, Vepuri S, Jadhav M, Soliman M, Govender T: Co-encapsulation of multi-lipids and polymers enhances the performance of vancomycin in lipid-polymer hybrid nanoparticles: In vitro and in silico studies.Mater Sci Eng C Mater Biol App/ 2016, 61:616-630.

17. Liu Y, Pan J, Feng SS: Nanoparticles of lipid monolayer shell and biodegradable polymer core for controlled release of paclitaxel: effects of surfactants on particles size, characteristics and in vitro performance.Int J Pharm 2010, 395:243-250.

18. Sonawane SJ, Kalhapure RS, Rambharose S, Mocktar C, Vepuri SB, Soliman M, Govender T: Ultrasmall lipid-dendrimer hybrid nanoparticles as a promising strategy for antibiotic delivery: In vitro and in silico studies.Int J Pharm 2016, 504:1-10.

19. Sabaeifard P, Abdi-Ali A, Soudi MR, Gamazo C, Irache JM: Amikacin loaded PLGA nanoparticles against Pseudomonas aeruginosa.European Journal of Pharmaceutical Sciences 2016, 93:392-398.

20. Dorati R, DeTrizio A, Spalla M, Migliavacca R, Pagani L, Pisani S, Chiesa E, Conti B, Modena T, Genta I: Gentamicin Sulfate PEG-PLGA/PLGA-H Nanoparticles: Screening Design and Antimicrobial Effect Evaluation toward Clinic Bacterial Isolates.Nanomaterials 2018, 8:37.

21. Liu Y, Shi L, Su L, van der Mei HC, Jutte PC, Ren Y, Busscher HJ: Nanotechnology-based antimicrobials and delivery systems for biofilm-infection control.Chemical Society Reviews 2019, 48:428-446.

22. Ghitman J, Biru El, Stan R, lovu H: Review of hybrid PLGA nanoparticles: Future of smart drug delivery and theranostics medicine.Materials \& Design 2020, 193:108805.

23. Sivadasan D, Sultan MH, Madkhali O, Almoshari Y, Thangavel N: Polymeric Lipid Hybrid Nanoparticles (PLNs) as Emerging Drug Delivery Platform-A Comprehensive Review of Their Properties, Preparation Methods, and Therapeutic Applications.Pharmaceutics 2021, 13:1291.

24. Fulaz S, Devlin H, Vitale S, Quinn L, O'Gara JP, Casey E: Tailoring Nanoparticle-Biofilm Interactions to Increase the Efficacy of Antimicrobial Agents Against Staphylococcus aureus.International journal of nanomedicine 2020, 15:4779-4791.

25. Sugano M, Morisaki H, Negishi Y, Endo-Takahashi Y, Kuwata H, Miyazaki T, Yamamoto M: Potential effect of cationic liposomes on interactions with oral bacterial cells and biofilms.J Liposome Res 2016, 26:156-162.

26. Arakha M, Pal S, Samantarrai D, Panigrahi TK, Mallick BC, Pramanik K, Mallick B, Jha S: Antimicrobial activity of iron oxide nanoparticle upon modulation of nanoparticle-bacteria interface.Scientific Reports 2015, 5:14813.

27. Da Costa D, Exbrayat-Héritier C, Rambaud B, Megy S, Terreux R, Verrier B, Primard C: Surface charge modulation of rifampicin-loaded PLA nanoparticles to improve antibiotic delivery in Staphylococcus aureus biofilms. Journal of Nanobiotechnology 2021, 19:12.

28. Wang DY, van der Mei HC, Ren Y, Busscher HJ, Shi L: Lipid-Based Antimicrobial Delivery-Systems for the Treatment of Bacterial Infections.Front Chem 2019, 7:872.

29. Latka A, Drulis-Kawa Z: Advantages and limitations of microtiter biofilm assays in the model of antibiofilm activity of Klebsiella phage KP34 and its depolymerase.Scientific Reports 2020, 
10:20338.

30. Fulaz S, Vitale S, Quinn L, Casey E: Nanoparticle-Biofilm Interactions: The Role of the EPS Matrix.Trends in Microbiology 2019, 27:915-926.

31. Habimana O, Semião AJC, Casey E: The role of cell-surface interactions in bacterial initial adhesion and consequent biofilm formation on nanofiltration/reverse osmosis membranes. Journal of Membrane Science 2014, 454:82-96.

32. Flemming H-C, Wingender J: The biofilm matrix.Nature Reviews Microbiology 2010, 8:623-633.

33. Wiegand I, Hilpert K, Hancock REW: Agar and broth dilution methods to determine the minimal inhibitory concentration (MIC) of antimicrobial substances.Nature Protocols 2008, 3:163-175.

\section{Tables}

Table 1. Optimization of LCHNPs based on vancomycin EE, LE, and $\zeta$-potential.

\begin{tabular}{cccccc}
\hline Sample & \multicolumn{2}{c}{ Independent variables } & \multicolumn{3}{c}{ Measured responses } \\
\cline { 2 - 6 } & Mass of PLGA (mg) & Mass of DOTAP $(\mathbf{m g})$ & $\begin{array}{c}\text { EE } \\
(\%)\end{array}$ & LE $(\mu \mathrm{g} / \mathbf{m g})$ & $\begin{array}{c}5 \text {-potential } \\
(\mathbf{m V})\end{array}$ \\
\hline LCHNP6:10 & 60 & 10 & $26.12 \pm 2.21$ & $49.34 \pm 3.78$ & $-9.58 \pm 1.22$ \\
\hline LCHNP120:10 & 120 & 10 & $44.19 \pm 3.42$ & $40.79 \pm 3.16$ & $-26.37 \pm 1.38$ \\
\hline LCHNP6:20 & 60 & 20 & $39.72 \pm 2.47$ & $59.58 \pm 3.70$ & $14.60 \pm 1.54$ \\
\hline LCHNP120:20 & 120 & 20 & $29.91 \pm 1.58$ & $25.64 \pm 1.35$ & $-4.59 \pm 0.47$ \\
\hline LCHNP45:20 & 45 & 20 & $48.66 \pm 0.42$ & $72.99 \pm 0.63$ & $36.13 \pm 0.31$ \\
\hline LCHNP30:20 & 30 & 20 & $5.14 \pm 0.68$ & $7.72 \pm 1.05$ & $13.86 \pm 2.11$ \\
\hline
\end{tabular}


Table 2. MIC and MBC of free- and encapsulated vancomycin against planktonic USA300.

\begin{tabular}{ccc}
\hline Sample & MIC $(\mu \mathrm{g} / \mathbf{m L})$ & MBC $(\mu \mathrm{g} / \mathbf{m L})$ \\
\hline Free-Van & 4 & 8 \\
\hline Van-PLGANPs & 7.36 & 14.72 \\
\hline Van-LCHNPs & 0.58 & 1.17 \\
\hline PLGANPs & $>10,000$ & $>10,000$ \\
\hline LCHNPs & $>10,000$ & $>10,000$ \\
\hline
\end{tabular}

Figures 

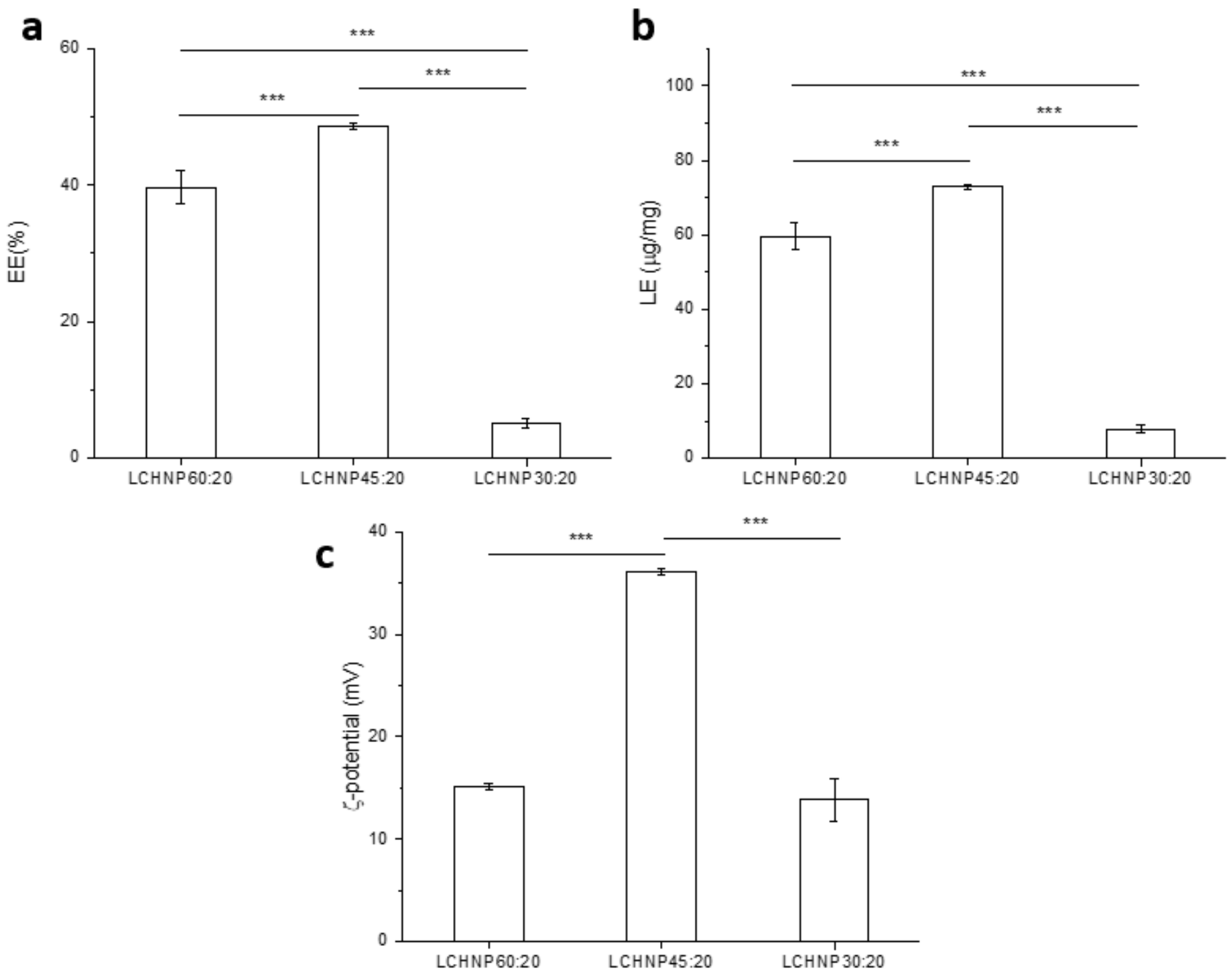

Figure 1

Bar plots of a) EE, b) LE, and c) Z-potential of LCHNP60:20, LCHNP45:20 and LCHNP30:20. Significant differences between groups were analyzed using Student's t-test on OriginPro 2021 (** $p<0.001, n=3$ ). 


\begin{tabular}{lllll}
\hline Sample & Hydrodynamic & -potential (mv) & EE\% & LE $(\mu \mathrm{g} / \mathrm{mg})$
\end{tabular}

\begin{tabular}{lllll}
\hline PLGANPs & $221.03 \pm 6.50$ & $-36.83 \pm 1.24$ & $24.11 \pm 0.64$ & $52.25 \pm 1.38$
\end{tabular}

\begin{tabular}{lllll}
\hline LCHNPs & $207.83 \pm 2.06$ & $36.13 \pm 0.31$ & $48.66 \pm 0.42$ & $72.99 \pm 0.63$
\end{tabular}
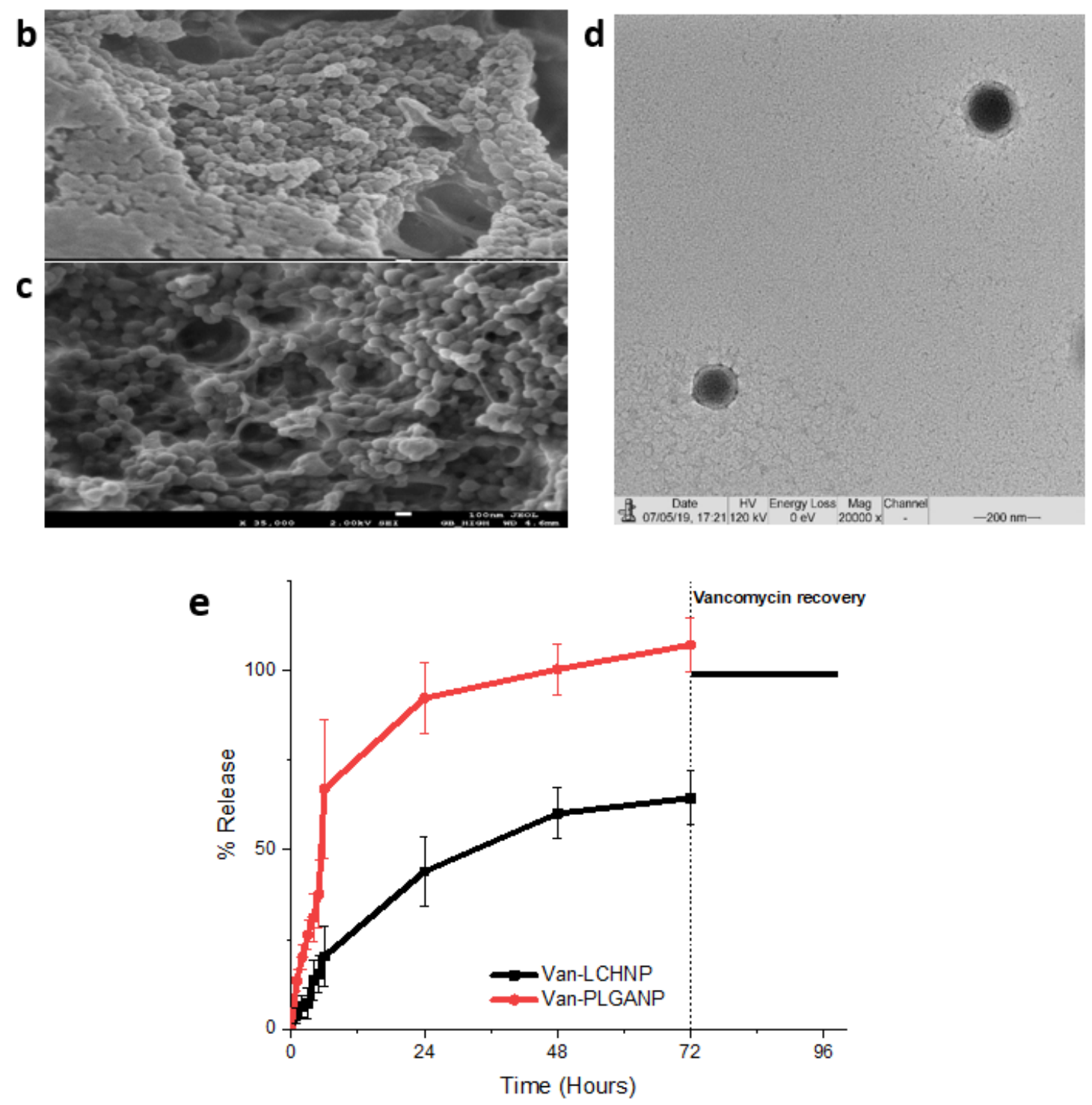

Figure 2

Comparison of optimized LCHNPs against bare PLGANPs. a) Summary of physicochemical characteristics (hydrodynamic diameter and Z-potential), EE and LE of PLGANPs and LCHNPs; b) FESEM micrograph of PLGANPs; c) FESEM micrograph of LCHNPs; d) TEM micrograph of LCHNPs showing outer coat of DOTAP on PLGA core; and e) vancomycin release profile of Van-PLGANPs and Van-LCHNPs. 

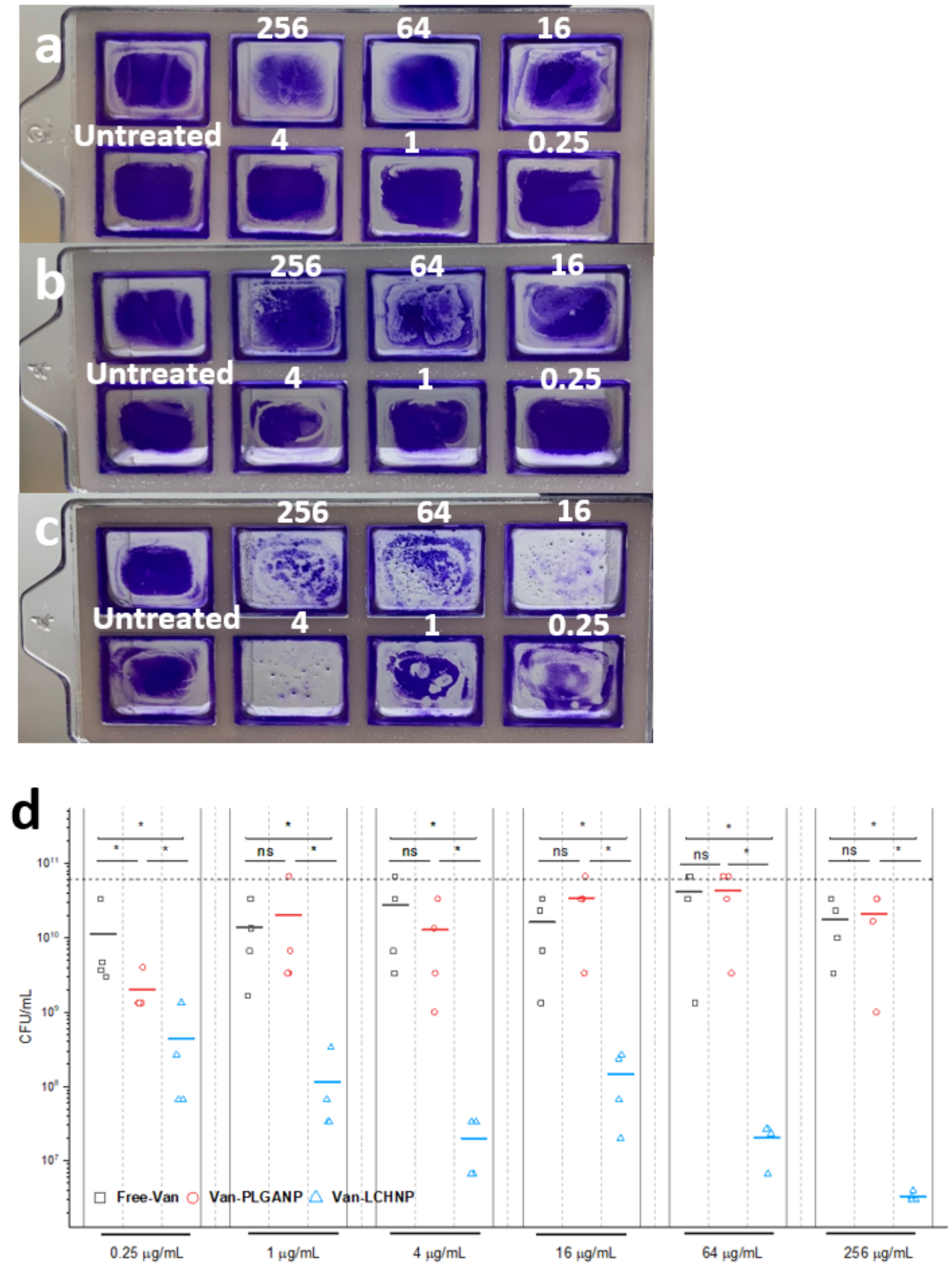

Figure 3

Assessment of the biofilm eradication capabilities of Free-Van, Van-PLGANPs and Van-LCHNPs. Crystal violet staining was performed post-treatment for visual observation of remaining biomass as pictured in a) Free-Van, b) Van-PLGANPs and c) Van-LCHNPs. The CFU counts that were derived from the remaining 
biofilm are shown in d); horizontal line at $\sim 2 \times 10^{10}$ denotes the CFU counts derived from untreated biofilm controls. Significant differences between groups were analyzed using Mann-Whitney Test on OriginPro 2021 ( $P<0.05, n=4)$.
a
$0.25 \mu \mathrm{g} / \mathrm{mL}$
$1 \mu \mathrm{g} / \mathrm{mL}$
$4 \mu \mathrm{g} / \mathrm{mL}$
$16 \mu \mathrm{g} / \mathrm{mL}$

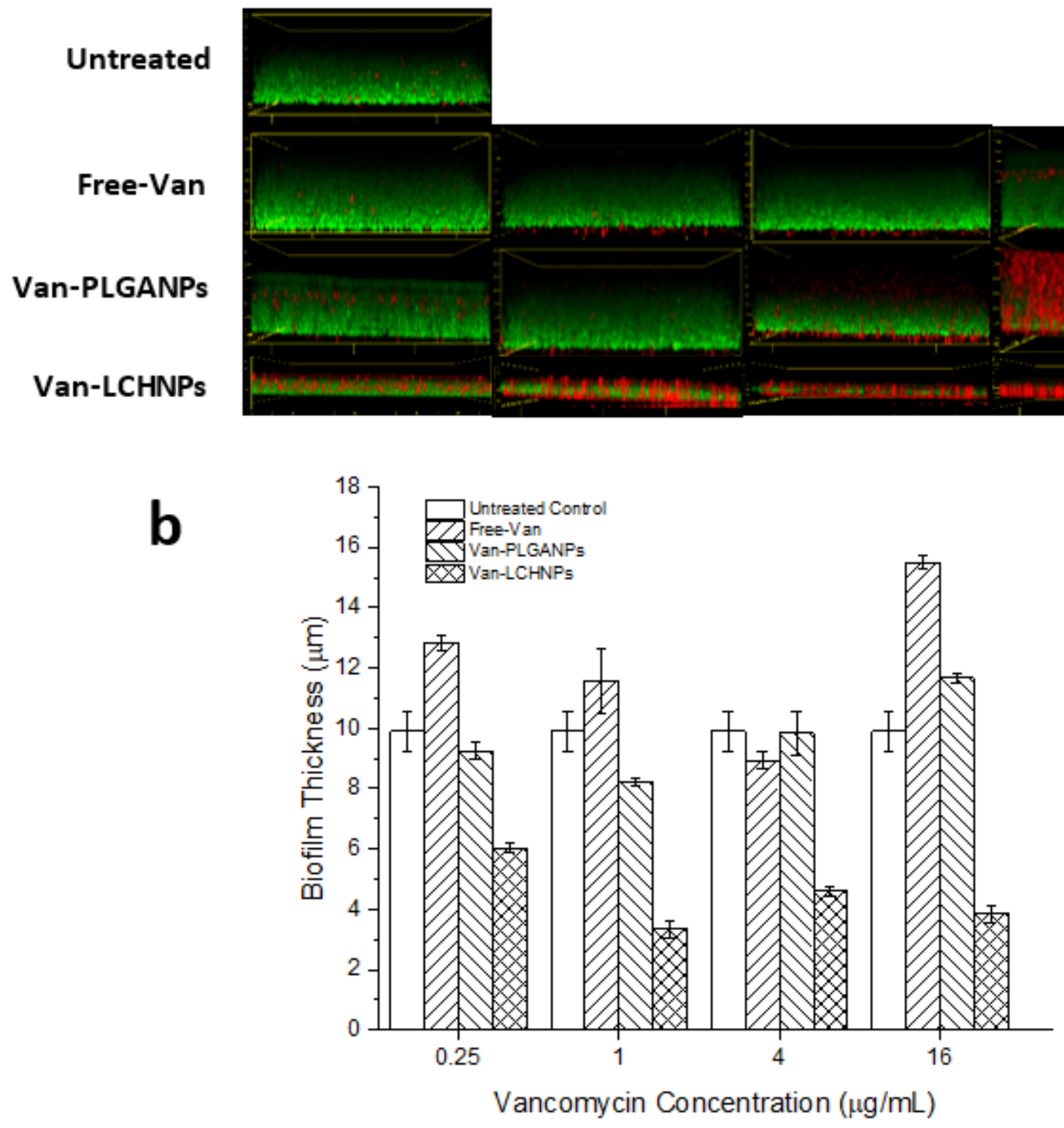

Figure 4

Observation of change in thicknesses of biofilms following treatment. a) Confocal images of each treatment group at vancomycin concentrations ranging from $0.25-16 \mu \mathrm{g} / \mathrm{mL}$. b) Measured thicknesses of biofilms following treatment. 


\section{DOTAP:PLGA}

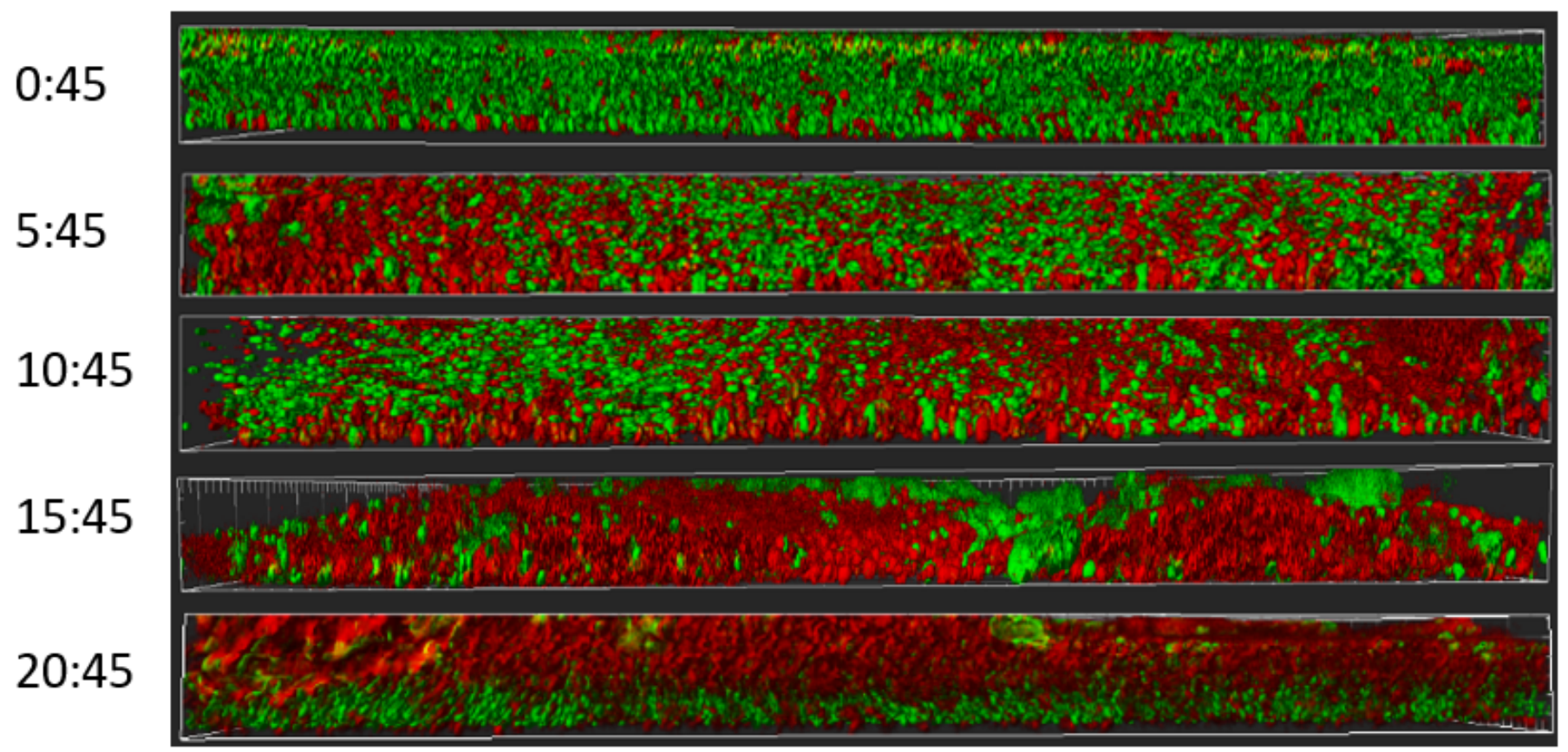

Figure 5

Confocal microscopy images showing the degrees of penetration of LCHNPs fabricated with varying ratios of DOTAP:PLGA.

\section{Supplementary Files}

This is a list of supplementary files associated with this preprint. Click to download.

- GraphicalAbstract.png 